\title{
Identification of hub genes associated with outcome of clear cell renal cell carcinoma
}

\author{
RENGUI LI ${ }^{1}$, LEI WANG ${ }^{1}$, XIAO WANG ${ }^{1}$, RONG-XIN GENG $^{2}$, NING LI $^{3}$ and XIU-HENG LIU ${ }^{1}$ \\ Departments of ${ }^{1}$ Urology, ${ }^{2}$ Neurosurgery and ${ }^{3}$ Cardiology, Renmin Hospital of Wuhan University, \\ Wuhan, Hubei 430060, P.R. China
}

Received April 10, 2019; Accepted December 5, 2019

DOI: $10.3892 / \mathrm{ol} .2020 .11389$

\begin{abstract}
Clear cell renal cell carcinoma (ccRCC) is one of the most common tumor types of the urinary system. Bioinformatics tools have been used to identify new biomarkers of ccRCC and to explore the mechanisms underlying development and progression of ccRCC. The present study analyzed the differentially expressed genes (DEGs) associated with RCC using data obtained from Gene Expression Omnibus datasets and GEO2R software. Gene Ontology (GO) and Kyoto Encyclopedia of Genes and Genomes (KEGG) pathway enrichment analysis of these DEGs was performed and analyzed using the Database for Annotation, Visualization and Integrated Discovery. A protein-protein interaction (PPI) network was constructed using the Search Tool for the Retrieval of Interacting Genes to identify the hub genes, defined as the genes with the highest degree of interrelation. Subsequently, differential expression and survival analyses of hub genes was performed using The Cancer Genome Atlas database and Gene Expression Profiling Interactive Analysis (GEPIA) online tool. Using GEO2R, 1,650 DEGs were identified, including 743 upregulated and 907 downregulated genes. GO and KEGG pathway analyses indicated that the upregulated DEGs were primarily involved in blood vessel and vasculature development, whereas downregulated DEGs were primarily involved in organic acid metabolic processes and carboxylic acid metabolic processes. Subsequently, important modules were identified in the PPI network using Cytoscape's Molecular Complex Detection. The 15 most connected hub genes were identified among DEGs, including glycine decarboxylase (GLDC), enolase 2 (ENO2) and topoisomerase II alpha. GEPIA revealed the association between expression levels of hub genes and survival. Specifically, GLDC and ENO2 were associated with the prognosis of patients with
\end{abstract}

Correspondence to: Professor Xiu-Heng Liu, Department of Urology, Renmin Hospital of Wuhan University, 238 Jiefang Road, Wuhan, Hubei 430060, P.R. China

E-mail: drliuxh@hotmail.com

Key words: clear cell renal cell carcinoma, bioinformatics analysis, protein-protein interaction, diagnosis, prognosis
RCC and thus, the effects of GLDC and ENO2 involvement in renal cancer were investigated in vitro. GLDC and ENO2 affected the proliferation and apoptosis of renal cancer cells. These hub genes may reveal a new mechanism underlying development or progression of RCC and identify new markers for its diagnosis and prognosis.

\section{Introduction}

Renal cell carcinoma (RCC) accounts for $2-3 \%$ of malignant tumors in adults (1). Notably, clear cell RCC (ccRCC) accounts for $70-85 \%$ of kidney cancer cases with an increasing incidence worldwide (2). Clinically, RCC can be divided into four stages according to the size of the tumor and extent of invasion and metastasis. The insidious onset of symptoms usually results in diagnosis in the first instance at an advanced stage of the disease (3). Owing to the resistance of ccRCC to radiotherapy and chemotherapy, the most effective treatment is radical or partial nephrectomy. However, the mortality rate of patients with metastatic RCC remains high (4). Targeted therapy with agents such as sorafenib and sunitinib have been used for metastatic RCC; however, its curative effects are limited (5). Thus, it is important to identify biomarkers to aid early diagnosis of RCC and to provide novel therapeutic targets.

Recently, a number of biomarkers for diagnosis and prognosis have been found and investigated. For example, the CXC chemokine receptor 4 (CXCR4), which is one of the most important markers of cancer stem cells, has been confirmed as a major chemokine receptor in solid tumors (6). A previous study demonstrated that CXCR4 may predict survival in patients with RCC. High-throughput sequencing is a common tool used in medical research in numerous types of cancer concerning early diagnosis, staging, grading and prognosis $(7,8)$. The use of bioinformatics to screen high-throughput sequencing allows identification of differentially expressed genes (DEGs) that may be associated with occurrence and development of certain diseases. In the present study, a Gene Expression Omnibus (GEO) dataset was selected and bioinformatics analysis was performed to identify the DEGs in ccRCC. Subsequently, the Search Tool for the Retrieval of Interacting Genes (STRING) was used to construct a protein-protein interaction (PPI) network and identify the hub genes in ccRCC. DEGs were analyzed to determine the biological process (BP), molecular 
function (MF) or cellular component (CC) associated with the genes using Kyoto Encyclopedia of Genes and Genomes (KEGG). A total of 15 hub genes were selected and overall survival analysis was performed to determine the relationship between each gene and survival of patients with ccRCC. The aim of the present study was to identify novel biomarkers and targets for the diagnosis and prognosis of RCC.

\section{Materials and methods}

Dataset. Data relevant to RCC was obtained from the GEO database and the GSE40435 (https://www.ncbi.nlm.nih. gov/geo/query/acc.cgi?acc=GSE40435) dataset was selected (9). This dataset was established on a GPL10558 platform. The GSE40435 dataset contains 101 pairs of ccRCC tumors and healthy adjacent tissue samples (age range, 42-84 years). In this database the basic information regarding each patient, including age, sex, tumor grade and type of tissue, is complete.

Differential gene expression analysis. The online software tool GEO2R (https://www.ncbi.nlm.nih. gov/geo/geo2r/?acc=GSE40435) was used to analyze the samples in the GSE40435 dataset. Using the GEO2R software, samples can be divided into two or more groups and the DEGs can be selected (10). A total of 202 samples were divided into two groups; the ccRCC tumor group and the adjacent non-tumor group. The Benjamini-Hochberg method was used to determine the false discovery rate (11) and the adjusted $\mathrm{P}$-value was used to reduce the likelihood of false positive errors. The selection criteria included an adjusted P-value of $<0.05$ and $\mid \log$ fold change (FC) $\geq 1$ (12).

Gene Ontology (GO) and KEGG pathway analysis of DEGs. $\mathrm{GO}$ analysis is a valuable approach to annotate genes and gene products, and assign characteristic biological properties to high-throughput genomic or transcriptome data (13). KEGG is a database collection which can be used to analyze genomes, biological pathways, diseases, chemical substances and drugs (14). DEGs selected by GEO2R were stratified according to whether they were upregulated or downregulated. Database for Annotation, Visualization and Integrated Discovery (DAVID; david.ncifcrf.gov/) was used for annotation of the GO and KEGG pathway results (15). $\mathrm{P}<0.05$ was used as a selection criterion for major BPs, MF and CC.

Establishing the PPInetwork and analyzing modules.STRING is an online application for evaluating PPI networks (16). STRING was used to map DEGs and identify potential interaction between DEGs. The selection criteria were: Confidence score $\geq 0.4$ and max number of interactors to show none/query proteins only $(17,18)$. Furthermore, the Molecular Complex Detection (MCODE) application in Cytoscape (version 3.6.1) was applied to select the PPI network modules, with a cutoff $=2$, node score cutoff $=0.2, \mathrm{k}$-core $=2$ and maximum depth $=100$ as the selection criteria (18). GO and KEGG pathway analyses of the selected modules were performed in DAVID to explore potential information. The network of 15 selected hub genes were visualized using STRING and the selection criteria were: Confidence score $\geq 0.4$ and a maximum number of interactors $\leq 5$. Fig. 1 shows a flow diagram of the methodology.
Comparing expression and survival. The differential gene expression in patients with RCC and patients without tumors was investigated using the standardized output of RNA sequencing data in The Cancer Genome Atlas and Genotype-Tissue Expression project databases (19). GEPIA was used to determine differences in the expression of hub genes between RCC tissues and non-tumorous tissues, and the results were visualized as box plots. Additionally, the Gene Expression Profiling Interactive Analysis (GEPIA; gepia.cancer-pku.cn/index.html) also detects changes in the survival curves caused by hub genes in RCC patients. University of California Santa Cruz Xena (xena.ucsc.edu/kaplan-survival-analysis/) was used to perform Kaplan-Meier survival analyses (20). Immunohistochemical data of patients with RCC and healthy individuals were obtained from the Human Protein Atlas (HPA) (21) to confirm the expression levels of the hub genes. For GLDC, the normal tissue was from a 61-year old man and the tumor was from a 59-years old woman. For ENO2, the normal tissue was from a 52-years old woman and the tumor was from a 52-years old woman. The GLDC expression level, age, sex and tumor stage of the 877 patients (age range, 28-90 years; mean age, 62 years) with renal cancer was obtained from the HPA (proteinatlas. org/ENSG00000178445-GLDC/pathology/renal+cancer) to explore the patient characteristics in the glycine decarboxylase (GLDC) high and GLDC low groups. After removal of incomplete data, complete information of 840 patients was gathered. Subsequently, these 840 patients were divided into two groups according to the level of GLDC expression $(n=420$ for each group). The relationship between the expression of GLDC and patient characteristics was then analyzed using $\chi^{2}$ test.

Gene set enrichment analysis (GSEA). RCC samples $(\mathrm{n}=101)$ from the GSE40435 dataset were divided into a high and low expression groups according to the median expression levels of GLDC and enolase 2 (ENO2). GSEA (version 3.0; software.broadinstitute.org/gsea/index.jsp) was used to examine the potential biological functions of GLDC and ENO2. The reference gene sets were annotated. Gene sets: c2.cp.kegg. v5.2.symbols.gmt, sets c2.cp.bp. v5.2.symbols.gmt, setsc2. cp.mf. v5.2.symbols.gmt and sets c2.cp.cc. v5.2.symbols.gmt. The cut-off criteria were $\mathrm{P}<0.05 \mathrm{P}<0.05$, enrichment score (ES) $>0.5$ and gene size $\geq 100(22,23)$.

Cell culture and transfection of small interfering (si)RNA and small activating ( $s a) R N A$. Human RCC cell line 786-O and normal renal tubular epithelial cell line HK-2 were obtained from The China Center for Type Culture Collection. The saRNA negative control and siRNA were purchased from Shanghai GenePharma Co., Ltd., and were used at the concentration of $40 \mathrm{nM}$. The siRNAs were transfected into cells using Lipofectamine ${ }^{\circledR} 2000$ (Invitrogen, Thermo Fisher Scientific, Inc.) for $48 \mathrm{~h}$ prior to subsequent experimentation. Cells were cultured at the density of $2 \times 10^{6}$ cells in RPMI-1640 media supplemented with $10 \%$ FBS (Gibco; Thermo Fisher Scientific, Inc.), $1 \%$ antibiotic solution (penicillin $100 \mathrm{U} / \mathrm{ml}$ and streptomycin $100 \mathrm{~g} / \mathrm{ml}$; Beyotime Institute of Biotechnology) at $37^{\circ} \mathrm{C}$ with $5 \% \mathrm{CO}_{2}$ in a humidified incubator. Before transfection, $5 \times 10^{4}$ cells were seeded into 6 -well plate. Sequences for siRNA targeting ENO2 were as follows: 5'-UUCUCUAUGGACAUG AUGGCU-3' guide, 5'-CCAUCAUGUCCAUAGAGAAGA-3' 


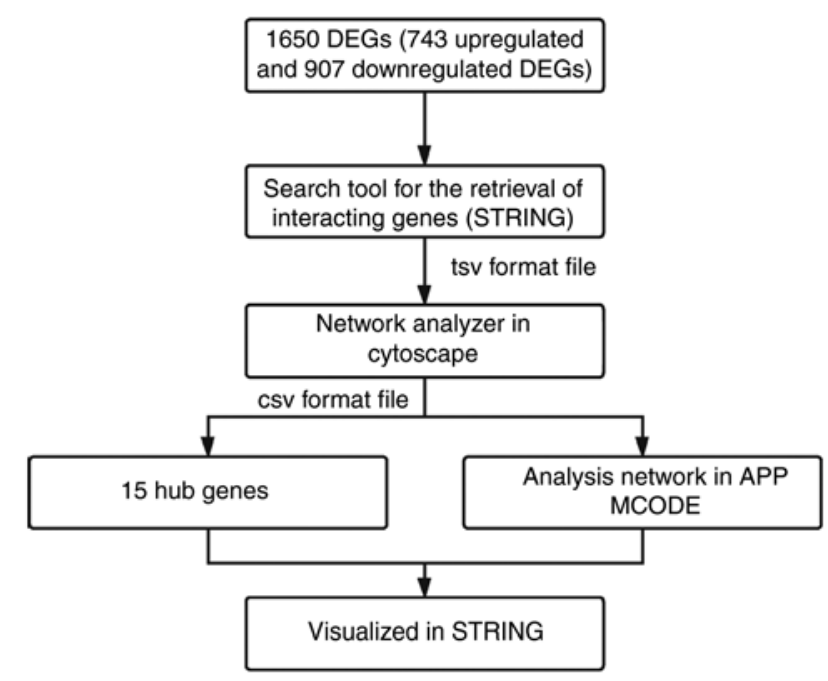

Figure 1. Flow diagram of PPI network construction and method used to analyze modules. DEGs, differentially expressed genes.

passenger. Sequences for saRNA targeting GLDC were as follows: 5'-AGUGUCUUGGUUGAGCGCA-3' guide; 5'-UGC GCUCAACCAAGACACU-3' passenger.

Cell proliferation assay. Cell viability was quantitatively evaluated using a Cell Counting kit-8 (CCK-8; Beyotime Institute of Biotechnology). After transfection by siRNA, saRNA and negative control, a total of $7 \times 10^{3}$ RCC cells/well were seeded into 96-well plates and exposed to a combination of different conditions. CCK-8 solution (10 $\mu \mathrm{l})$ was added to each well for $2 \mathrm{~h}$ at $37^{\circ} \mathrm{C}$, after which the optical density was estimated by measuring the absorbance at $450 \mathrm{~nm}$ using a microplate reader (Victor 31420 Multilabel Counter; PerkinElmer, Inc.).

Western blotting. RCC cells were cultured in 6-well plates for $48 \mathrm{~h}$ and transfected with siRNA or shRNA. Cells were lysed with RIPA buffer containing protease inhibitors (Beyotime Institute of Biotechnology. A bicinchoninic acid protein assay kit (Beyotime Institute of Biotechnology) was used to determine the concentration of protein. Proteins $(25 \mu \mathrm{g})$ were separated by $10 \%$ SDS-PAGE and subsequently transferred to PVDF membranes (EMD Millipore). The membranes were blocked in 5\% non-fat dry milk in TBS. Subsequently, the membranes were incubated with a rabbit or mouse primary antibody against GLDC (1:1,000; Abcam; cat. no. ab232989), ENO2 (1:1,000; Abcam; cat. no. ab189891), Bax (1:1,000; Abcam; cat. no. ab32503) and Bcl-2 (1:1,000; Abcam; cat. no. ab32124) at $4^{\circ} \mathrm{C}$ overnight. After washing with TBS-Tween 20 three times (10 $\mathrm{min} /$ wash), the membranes were incubated with the relevant secondary antibody for $1 \mathrm{~h}$ at room temperature, followed by three washes with TBS-Tween 20 in the dark. The membranes were scanned using a two-color Odyssey infrared imaging system (LI-COR Biosciences). Protein expression levels were normalized to those of GAPDH from the same membrane. Densitometry analysis was performed using ImageJ software (version 1.52r; National Institutes of Health).
V-PE/7-AAD kit (MultiSciences) and Annexin-V-FITC/PI kit (BD Biosciences) were used to quantify the percentage of apoptotic cells using a flow cytometer (FACSCalibur; BD Biosciences). FlowJo v10.6.1 software (FlowJo, LLC) was used for analysis. Cells were seeded into 6-well plates after being transfected for $48 \mathrm{~h}$ with siRNA or saRNA, NC and control. Adherent cells were collected and co-stained with $5 \mu$ l Annexin $\mathrm{V}-\mathrm{PE}$ and $5 \mu \mathrm{l}$ 7-AAD for $15 \mathrm{~min}$ at room temperature in the dark prior to flow cytometry analysis. Live cells were cells fluorescing positively for both PE and 7-AAD negative, early apoptotic cells were cells fluorescing with PE alone, necrotic cells did not show fluorescence for either fluorophore and late apoptotic and dead cells showed 7-AAD fluorescence alone.

Hoechst 33258 apoptosis assay. Hoechst 33258 Staining kit (Beyotime Institute of Biotechnology) was used to detect apoptotic morphological features. A total of $1 \times 10^{5}$ cells/well in the exponential growth phase were seeded into a 6 -well plate. Cells were cultured for $24 \mathrm{~h}$ and stained with Hoechst 33258 at room temperature for $15 \mathrm{~min}$. Apoptotic morphological features (chromatin condensation, nuclear fragmentation) were observed and captured using a fluorescent microscope (BX51; Olympus Corporation; magnification, x200).

Statistical analysis. Data analysis was performed in GraphPad prism version (GraphPad Software, Inc.). Data are presented as the mean \pm standard error of mean of 3 repeats. An ANOVA with a post-hoc Tukey's test was used to compare the differences between multiple groups. $\mathrm{P}<0.05$ was considered to indicate a statistically significant difference.

\section{Results}

DEGs and hub genes. The GSE40435 dataset contains 202 samples, including 101 pairs of ccRCC tumors and adjacent healthy tissue. A total of 1,650 DEGs (743 upregulated and 907 downregulated) were selected. The volcano map (Fig. 2A) and the heat map (Fig. 2B) graphically visualize the selected DEGs with $\mathrm{P}<0.05$ and $\mid \log \mathrm{FCl} \geq 1$. Subsequently, the 15 most relevant hub genes were selected according to the degree of connectivity in PPI networks (Table I).

GO and KEGG pathway enrichment analyses. GO and KEGG pathway enrichment analyses were performed by inputting upregulated and downregulated DEGs into DAVID to determine their relationship and study the functions of the DEGs (Fig. 3). Fig. 3A and B show the results of GO enrichment analysis. The upregulated DEGs were enriched in BPs, including 'blood vessel development', 'vasculature development', 'angiogenesis', 'locomotion' and 'extracellular matrix organization'. The downregulated DEGs were enriched in BPs, including 'organic acid metabolic process', 'carboxylic acid metabolic process', 'oxoacid metabolic process', 'organic acid catabolic process' and 'small molecule catabolic process'. For MF, the upregulated DEGs were primarily enriched in 'receptor binding', 'identical protein binding', 'cargo receptor activity', 'protein homodimerization activity' and 'extracellular matrix binding'. The downregulated DEGs were enriched in 'cofactor binding', 'anion binding', 'active transmembrane transporter activity', 'secondary active transmembrane transporter 


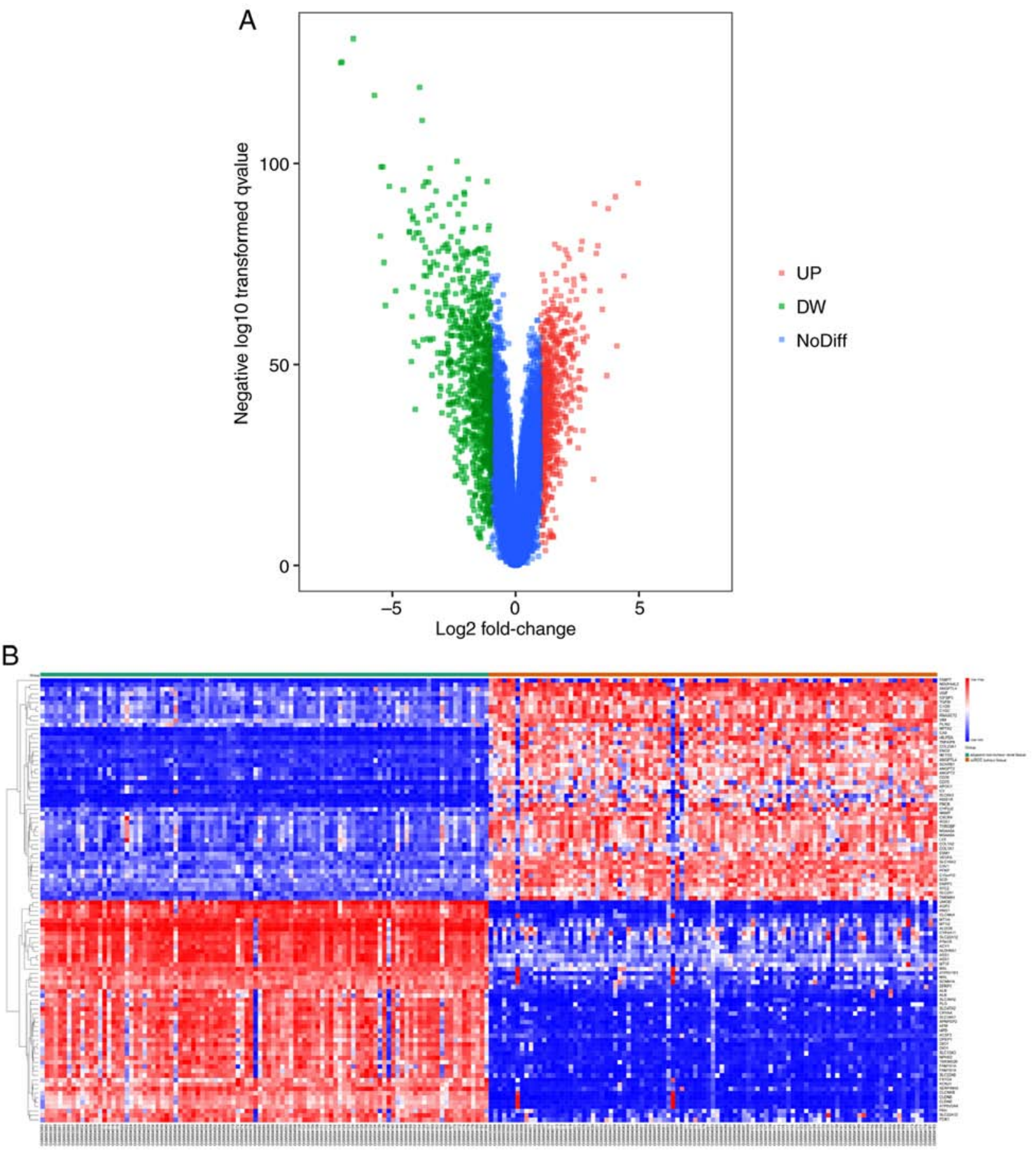

B

Figure 2. DEGs in renal cancer. (A) Volcano plot of DEGs (fold change $\geq 1$ and P-value $\leq 0.05$ ). Blue dots represent statistically significant differentially expressed genes. Green dots represent downregulated genes and red dots represent upregulated genes. (B) Heatmap of 100 differentially expressed genes in renal cell carcinoma including 50 upregulated genes and 50 downregulated genes. Blue dots represent downregulated genes and red dots represent up-regulated genes. DEG, differentially expressed genes; UP, upregulated; DW, downregulated; NoDiff, no difference.

activity' and 'carboxylic acid binding' (Table II). For CC, the upregulated DEGs were enriched in 'extracellular space', 'collagen trimer', 'extracellular region', 'extracellular region part' and 'proteinaceous extracellular matrix'. Downregulated DEGs were enriched in 'extracellular exosome', 'extracellular vesicle', 'extracellular organelle', 'membrane-bounded vesicle' and 'extracellular region part' (Table II).

The upregulated DEGs were enriched in 'Focal adhesion', the 'PI3K-Akt signaling pathway', 'ECM-receptor interaction', the HIF-1 signaling pathway' and 'Staphylococcus aureus infection'.
The downregulated DEGs were enriched in 'Metabolic pathways', 'Biosynthesis of antibiotics', 'Glycine, serine and threonine metabolism', 'Mineral absorption' and 'Aldosterone-regulated sodium reabsorption' (Fig. 3C; Table III).

Screening hub genes and three modules from the PPI network. The PPI network constructed using hub genes revealed the correlation between DEGs, where hub genes with higher degrees of correlation were detected using Cytoscape. The following hub genes were selected: Albumin; vascular 
Table I. Top 15 hub genes with highest degree of connectivity.

\begin{tabular}{lcc}
\hline Gene & Degree of connectivity & Adjusted P-value \\
\hline ALB & 222 & $3.05 \times 10^{-56}$ \\
VEGFA & 150 & $1.39 \times 10^{-58}$ \\
TOP2A & 141 & $5.15 \times 10^{-53}$ \\
EGFR & 114 & $2.73 \times 10^{-32}$ \\
EGF & 111 & $5.01 \times 10^{-60}$ \\
EHHADH & 102 & $7.34 \times 10^{-17}$ \\
MYC & 100 & $2.62 \times 10^{-49}$ \\
CD44 & 97 & $6.41 \times 10^{-30}$ \\
GLDC & 95 & $1.37 \times 10^{-67}$ \\
ALDH7A1 & 84 & $3.05 \times 10^{-52}$ \\
ENO2 & 83 & $7.62 \times 10^{-73}$ \\
CSF1R & 81 & $1.43 \times 10^{-43}$ \\
ALDH3A2 & 81 & $8.34 \times 10^{-29}$ \\
ALDH1B1 & 79 & $1.89 \times 10^{-49}$ \\
ALDH4A1 & 79 & $4.13 \times 10^{-56}$ \\
\hline
\end{tabular}

endothelial growth factor-A; topoisomerase II a; epidermal growth factor receptor (EGFR); EGF; enoyl-CoA hydratase and 3-hydroxyacyl CoA dehydrogenase; MYC; CD44; GLDC; aldehyde dehydrogenase 7 family member A1; ENO2; colony stimulating factor 1 receptor; aldehyde dehydrogenase 3 family member A2; aldehyde dehydrogenase 1 family member B1; and aldehyde dehydrogenase 4 family member A1. Subsequently, a PPI network of the 15 most relevant hub genes was constructed (Fig. 4A) and the top four modules were identified using the MCODE application in Cytoscape (Fig. 4B-E). These four modules were associated with valine, leucine and isoleucine degradation, retinol metabolism, glycolysis and gluconeogenesis (Table IV).

Differential expression and survival curves of hub genes. The GEPIA online tool revealed the upregulated and downregulated expression of genes between RCC and non-tumorous tissues (Fig. 5A and B). Expression levels of GLDC mRNA were significantly decreased in RCC compared with normal tissues (Fig. 5A), whereas ENO2 mRNA expression levels were significantly increased in renal carcinoma compared with normal tissues (Fig. 5B). In addition, immunohistochemical data of patients with RCC and normal patients were analyzed using HPA, and the results demonstrated that GLDC expression was low in tumor whereas ENO2 expression was high in tumor (Fig. 5C and D). KM survival curves indicated that GLDC may inhibit tumor development and ENO2 may promote tumor progression (Fig. 5E and F). GEPIA and UCSC Xena were performed, demonstrating that low expression levels of GLDC in RCC was associated with a less favorable prognosis $(\mathrm{P}=0.0082$ and $\mathrm{P}=0.02541$, respectively $)$. Analyses of ENO2 revealed that high expression levels of ENO2 was associated with poor prognosis (GEPIA, $\mathrm{P}=0.02$; UCSC Xena, $\mathrm{P}=0.01375$ ). CIs and $\log$-rank $\mathrm{P}$-value were calculated as shown in the plot (Fig. 5E and F). The relationship between the expression levels of GLDC and patient characteristics were analyzed, demonstrating that GLDC expression levels are significantly associated with patient age and disease stage, whereas no significant difference in terms of sex were observed (Table V).

GSEA. GSEA was performed to relate the hub genes to the GO analysis and KEGG pathway database and explore the function of these genes. A total of four functional gene sets were enriched. The gene sets enriched in RCC with high expression of GLDC were 'ribosomal subunit' and 'structural constituent of ribosome'. These two gene sets were primarily involved in cell proliferation and differentiation pathways (Fig. 5G). The gene sets enriched in RCC with high ENO2 expression levels were 'establishment of protein localization to endoplasmic reticulum' and 'protein localization to endoplasmic reticulum' (Fig. 5H). These gene sets were focused on pathways associated with cell apoptosis and endoplasmic reticulum stress.

GLDC acts as a tumor suppressor gene, whereas ENO2 is an oncogene in RCC. The expression levels of GLDC and ENO2 were examined in the normal renal tubular epithelial cell line HK-2 and the renal clear cell carcinoma cell line 786-O. It was found that GLDC had reduced expression levels in 786-O cells, whereas ENO2 was highly expressed in 786-O cells. saRNA was used to induce GLDC overexpression in 786-O cells and siRNA was used to knockdown ENO2 expression (Fig. 6A). There was a significant decrease in the proliferative capacity of the sa-GLDC-786-O and si-ENO2-786-O cells after 72 h compared to control group ( $\mathrm{P}<0.0001$; Fig. 6B). An Annexin V-PE/7-AAD double staining assay was performed detect cell apoptosis using flow cytometry. The number of apoptotic 786-O cells was increased in sa-GLDC-786-O cells and si-ENO2-786-O cells compared to control group (Fig. 6C) and the results of Hoechst 33258 staining assay were consistent with Annexin V-PE/7-AAD double staining assay (Fig. 6D and E). Finally, it was demonstrated that the expression of Bax increased and expression of Bcl-2 decreased (Fig. 6F). These data showed that GLDC promoted and ENO2 inhibited apoptosis of renal cancer cells, which suggests that GLDC acts as a tumor suppressor gene whereas ENO2 acts as an oncogene in RCC.

\section{Discussion}

A tumor is a manifestation of a disease dependent on a number of genes and its formation is associated with a variety of factors, including metabolic capacity and immune regulation. The essential attribute of a tumor is the loss of cell cycle regulation control, resulting in uncontrollable cell proliferation (24). The reasons for this may be the activation of one or more proto-oncogenes and mutations of or deletions in tumor suppressor genes (25). Genetic deletion mutations can lead to the inability of the immune system to recognize tumors, leading to immune escape (26). Therefore, changes in gene expression levels in cancer are particularly important.

The insidious onset of symptoms usually results in delayed diagnosis at an advanced stage of RCC (27). Therefore, the identification of specific biomarkers to aid diagnosis of RCC and effective therapeutic targets is needed. In the present study, the GSE40435 dataset, containing 101 pairs of tumors and adjacent healthy tissue, was analyzed. Although the 
A

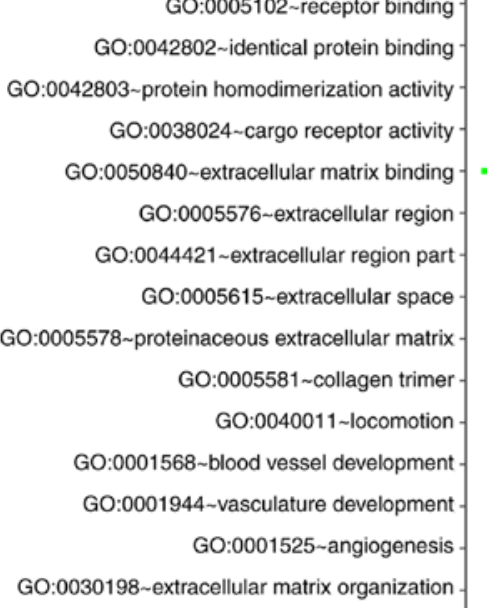

G0:0042802 identical protein binding

G0:0042803 protein homodimerization activity

GO:0038024 cargo receptor activity

GO:0050840 extracellular matrix binding - .

GO:0005576 extracellular region

GO:0005615 extracellular space

GO:0005578 proteinaceous extracellular matrix

GO:0005581 collagen trimer

GO:0040011 locomotion

GO:0001568 blood vessel development

GO:0001944 vasculature development

GO:0001525 angiogenesis

GO:0030198 extracellular matrix organization

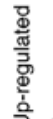

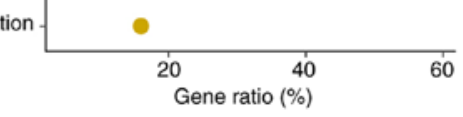

B

GO:0022804 active transmembrane transporter activity- = GO:0048037 cofactor binding. = GO:0043168 anion binding- =

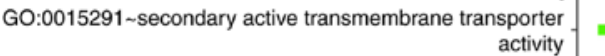

GO:0031406 carboxylic acid binding -

GO:0044421 extracellular region part

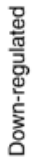

C


GO:0031988 membrane-bounded vesicle

GO:0070062 extracellular exosome

GO:1903561 extracellular vesicle

GO:0043230 extracellular organelle GO:0006082 organic acid metabolic process GO:0019752 carboxylic acid metabolic process

GO:0043436 0xoacid metabolic process GO:0044282 small molecule catabolic process

GO:0016054 organic acid catabolic process

10

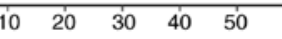

Gene ratio (\%)

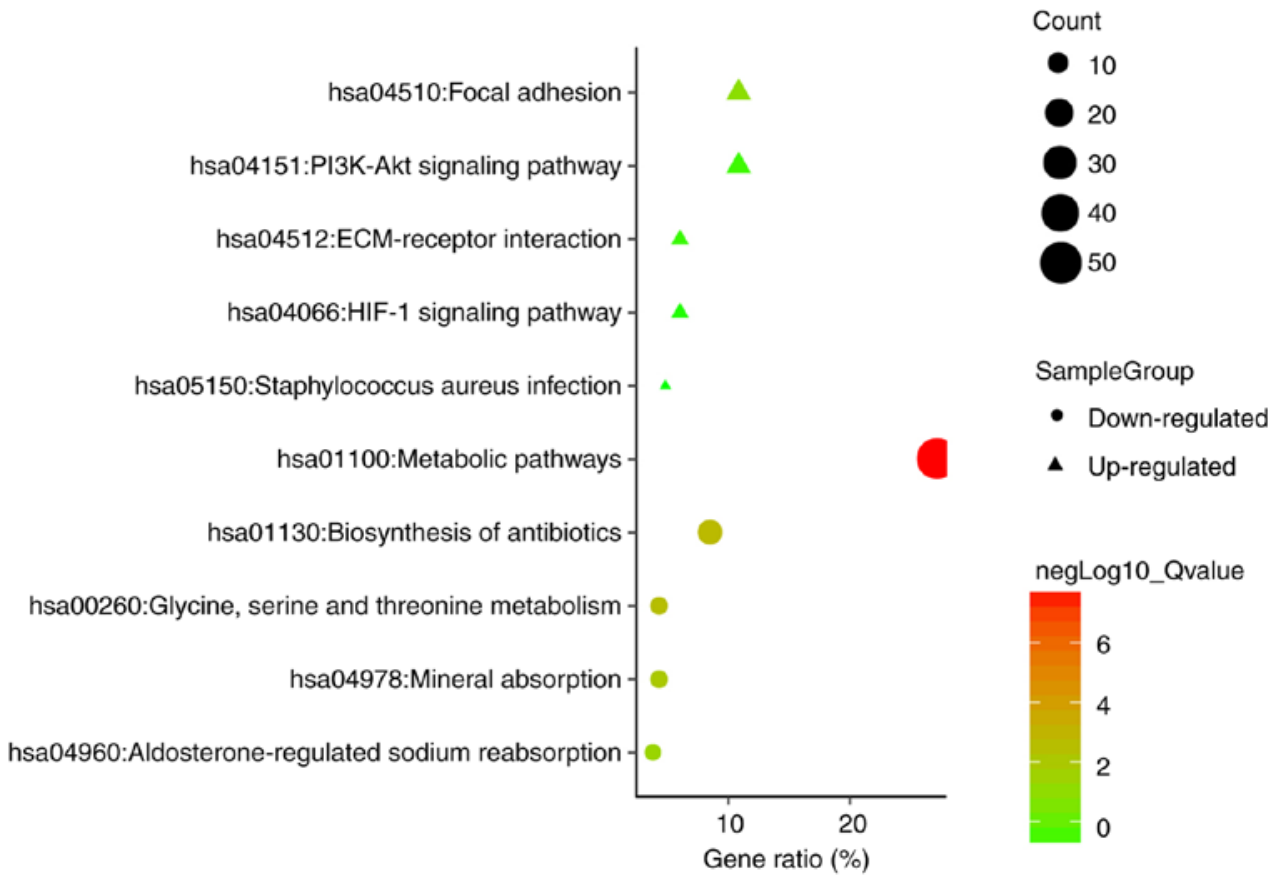

Gene ratio (\%)
SampleGroup

- GOTERM_BP_FAT

- GOTERM_CC_fat

- GOTERM_MF_FAT

Count

- 10

20

30

40

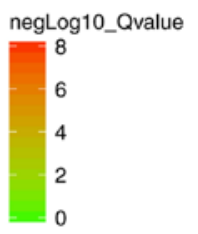

SampleGroup

- goterm_BP_fat

- goterm_CC_fat

- GOTERM_MF_FAT

Count

- 25

50

75

negLog10_Qvalue

20

10

Figure 3. Results of GO analysis. (A) upregulated and (B) downregulated DEGs enriched in BP, MF and CC. (C) KEGG pathway analysis showed the most significant pathway of up/downregulated DEGs. GO, Gene Ontology; BP, Biological Processes; MF, Molecular Function; CC, Cellular Component; KEGG, Kyoto Encyclopedia of Genes and Genomes 
Table II. Gene ontology analysis of differentially expressed genes associated with renal cell carcinoma.

A, Upregulated genes

\begin{tabular}{|c|c|c|c|c|}
\hline Category & Count & Ratio & P-value & FDR \\
\hline \multicolumn{5}{|l|}{$\mathrm{BP}$} \\
\hline GO:0001568-blood vessel development & 19 & 22.89157 & $5.91 \times 10^{-11}$ & $1.05 \times 10^{-07}$ \\
\hline GO:0001944-vasculature development & 19 & 22.89157 & $1.50 \times 10^{-10}$ & $2.68 \times 10^{-07}$ \\
\hline GO:0001525-angiogenesis & 15 & 18.07229 & $3.79 \times 10^{-09}$ & $6.76 \times 10^{-06}$ \\
\hline GO:0040011-locomotion & 25 & 30.12048 & $2.81 \times 10^{-08}$ & $5.02 \times 10^{-05}$ \\
\hline GO:0030198-extracellular matrix organization & 13 & 15.66265 & $2.94 \times 10^{-08}$ & $5.23 \times 10^{-05}$ \\
\hline \multicolumn{5}{|l|}{$\mathrm{CC}$} \\
\hline GO:0005615-extracellular space & 31 & 37.3494 & $2.51 \times 10^{-12}$ & $3.19 \times 10^{-09}$ \\
\hline GO:0005581-collagen trimer & 10 & 12.04819 & $1.94 \times 10^{-09}$ & $2.47 \times 10^{-06}$ \\
\hline GO:0005576-extracellular region & 49 & 59.03614 & $2.58 \times 10^{-09}$ & $3.29 \times 10^{-06}$ \\
\hline GO:0044421-extracellular region part & 44 & 53.01205 & $6.54 \times 10^{-09}$ & $8.32 \times 10^{-06}$ \\
\hline GO:0005578-proteinaceous extracellular matrix & 14 & 16.86747 & $2.49 \times 10^{-08}$ & $3.17 \times 10^{-05}$ \\
\hline \multicolumn{5}{|l|}{ MF } \\
\hline GO:0005102-receptor binding & 23 & 27.71084 & $3.23 \times 10^{-07}$ & $4.53 \times 10^{-04}$ \\
\hline GO:0042802-identical protein binding & 20 & 24.09639 & $7.57 \times 10^{-06}$ & 0.010638 \\
\hline GO:0038024-cargo receptor activity & 5 & 6.024096 & $2.81 \times 10^{-04}$ & 0.39348 \\
\hline GO:0042803-protein homodimerization activity & 11 & 13.25301 & 0.001746 & 2.425067 \\
\hline GO:0050840-extracellular matrix binding & 4 & 4.819277 & 0.001839 & 2.551809 \\
\hline
\end{tabular}

B, Downregulated

\begin{tabular}{|c|c|c|c|c|}
\hline Category & Count & Ratio & P-value & FDR \\
\hline \multicolumn{5}{|l|}{$\mathrm{CC}$} \\
\hline GO:0070062-extracellular exosome & 107 & 54.87179 & $3.25 \times 10^{-33}$ & $4.34 \times 10^{-30}$ \\
\hline GO:1903561-extracellular vesicle & 107 & 54.87179 & $5.11 \times 10^{-33}$ & $6.83 \times 10^{-30}$ \\
\hline GO:0043230-extracellular organelle & 107 & 54.87179 & $5.27 \times 10^{-33}$ & $7.06 \times 10^{-30}$ \\
\hline GO:0031988-membrane-bounded vesicle & 108 & 55.38462 & $2.53 \times 10^{-24}$ & $3.39 \times 10^{-21}$ \\
\hline GO:0044421-extracellular region part & 110 & 56.41026 & $5.33 \times 10^{-23}$ & $7.13 \times 10^{-20}$ \\
\hline \multicolumn{5}{|l|}{ MF } \\
\hline GO:0048037-cofactor binding & 18 & 9.230769 & $1.71 \times 10^{-08}$ & $2.57 \times 10^{-05}$ \\
\hline GO:0043168-anion binding & 18 & 9.230769 & $2.91 \times 10^{-08}$ & $4.37 \times 10^{-05}$ \\
\hline $\begin{array}{l}\text { GO:0022804-active transmembrane transporter } \\
\text { activity }\end{array}$ & 20 & 10.25641 & $4.68 \times 10^{-08}$ & $7.04 \times 10^{-05}$ \\
\hline $\begin{array}{l}\text { GO:0015291-secondary active transmembrane } \\
\text { transporter activity }\end{array}$ & 16 & 8.205128 & $8.44 \times 10^{-08}$ & $1.27 \times 10^{-04}$ \\
\hline GO:0031406-carboxylic acid binding & 12 & 6.153846 & $3.06 \times 10^{-06}$ & 0.004593 \\
\hline
\end{tabular}

GO, Gene Ontology; BP, biological processes; MF, molecular function; CC, cell component; FDR, false discovery rate.

number of samples included in the present study is limited, the data mining performed may be valuable. In gastric cancer, Sun et al (28) utilized the gene expression profile of GSE54129 containing only 111 gastric cancer samples and 21 healthy gastric mucosa epithelium. Chen et al (29) used bioinformatic analysis to demonstrate that the COP9 signalosome subunit 7B may be a prognostic marker and therapeutic target in renal cancer. Among differentially expressed genes, Wan et al (30) identified Aurora kinase B as a marker for predicting the prognosis of renal cancer. In the present study, the prognostic value of GLDC and ENO2 in RCC were assessed and it was hypothesized that they may be associated with cancer and carcinogenicity. DEGs were identified using bioinformatics and the 15 most important genes among these were determined using PPI network analysis. In these 15 genes, altered expression levels of GLDC and ENO2 exerted a significant effect on the prognosis of patients with renal cancer. Finally, GSEA was used to identify the BPs which may have been 


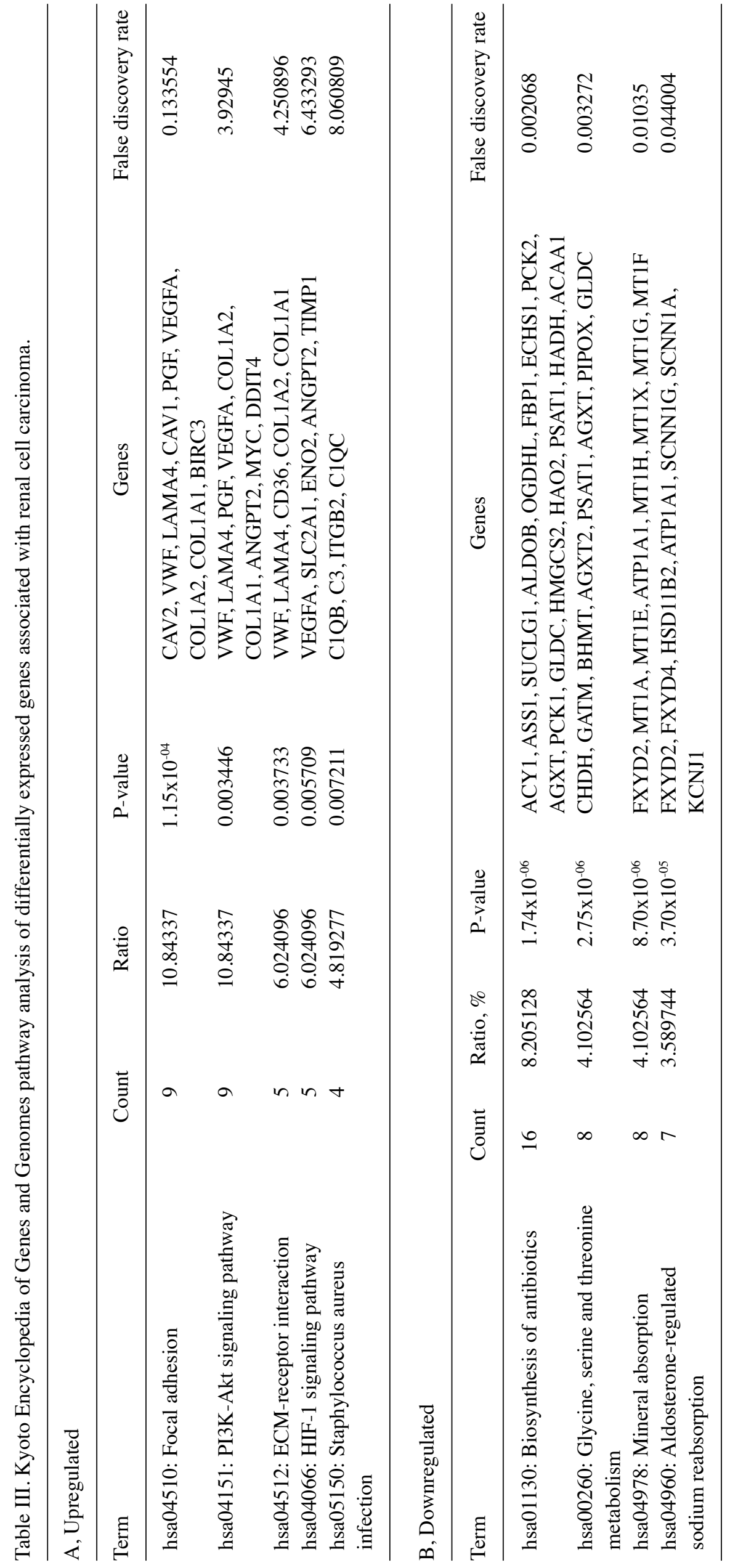


A

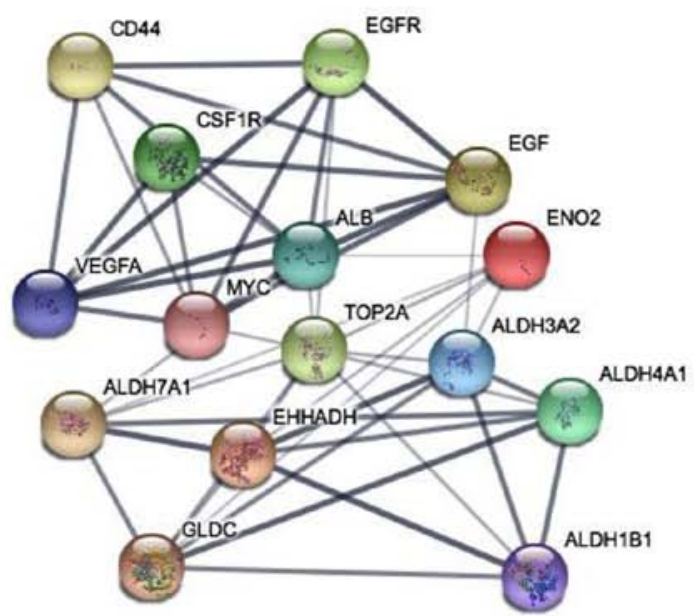

B

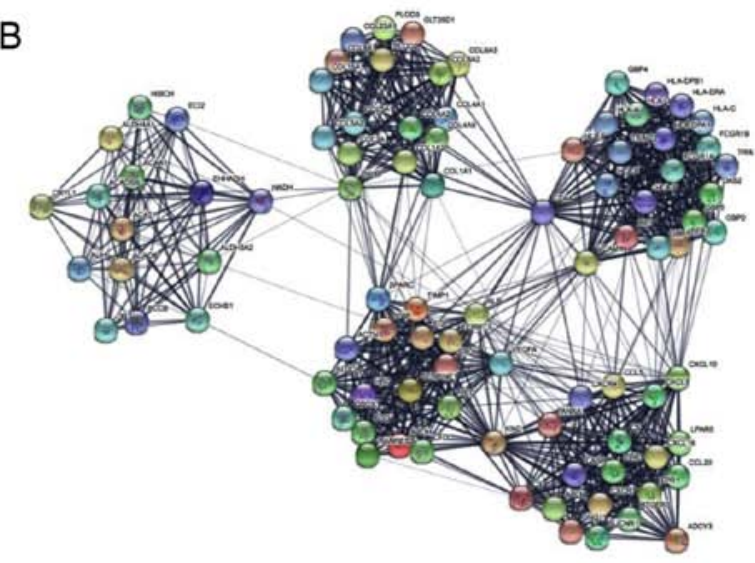

D

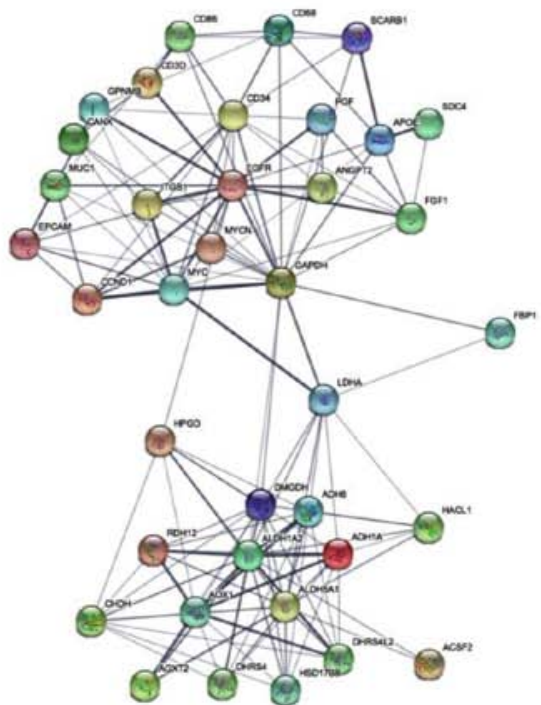

C

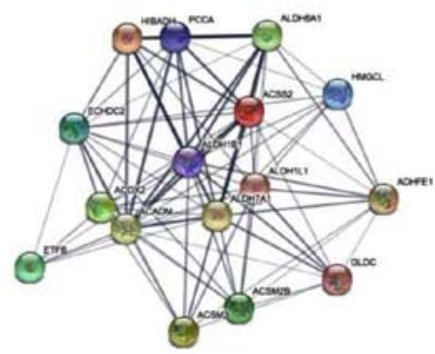

E

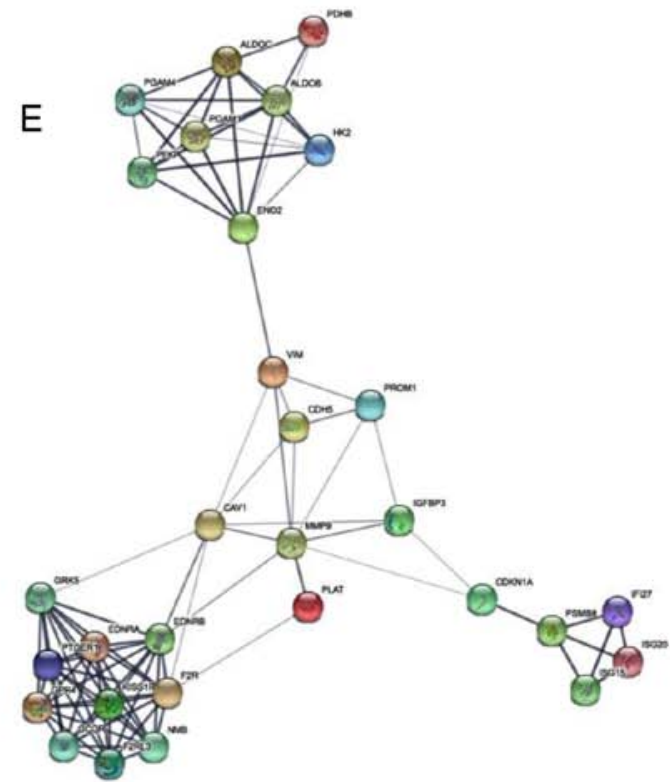

Figure 4. PPI networks of DEGs (A) PPI network of top 15 hub genes. (B, C, D, E) Top 4 modules from the PPI network. (B) module 1: Score=20.659, (C) module 2: Score=10.8, (D) module 3: Score=8.056, (E) module 4: Score=6.69. PPI, protein-protein interaction.

affected by altered expression levels of GLDC and ENO2. An understanding of the biological mechanisms regulated by GLDC and ENO2 in RCC may have research value.

GLDC, also termed glycine cleavage system $P$ protein and HYGN1, is involved in nonketotic hyperglycinemia $(31,32)$. Diseases associated with GLDC include glycine encephalopathy and neonatal glycine encephalopathy (33). Metabolism, glyoxylate metabolism and glycine degradation are among the pathways related to GLDC. GLDC has been shown to serve an important role in certain types of cancer, such as gastric cancer (34), breast cancer (35) and lung cancer (36); however, to the best of our knowledge, there has been no research investigating the relationship between GLDC and RCC. In lung cancer, GLDC is an essential oncogene promoting tumorigenesis through its metabolic action (37). In gastric cancer, GLDC promoter hypermethylation regulates transcriptional 


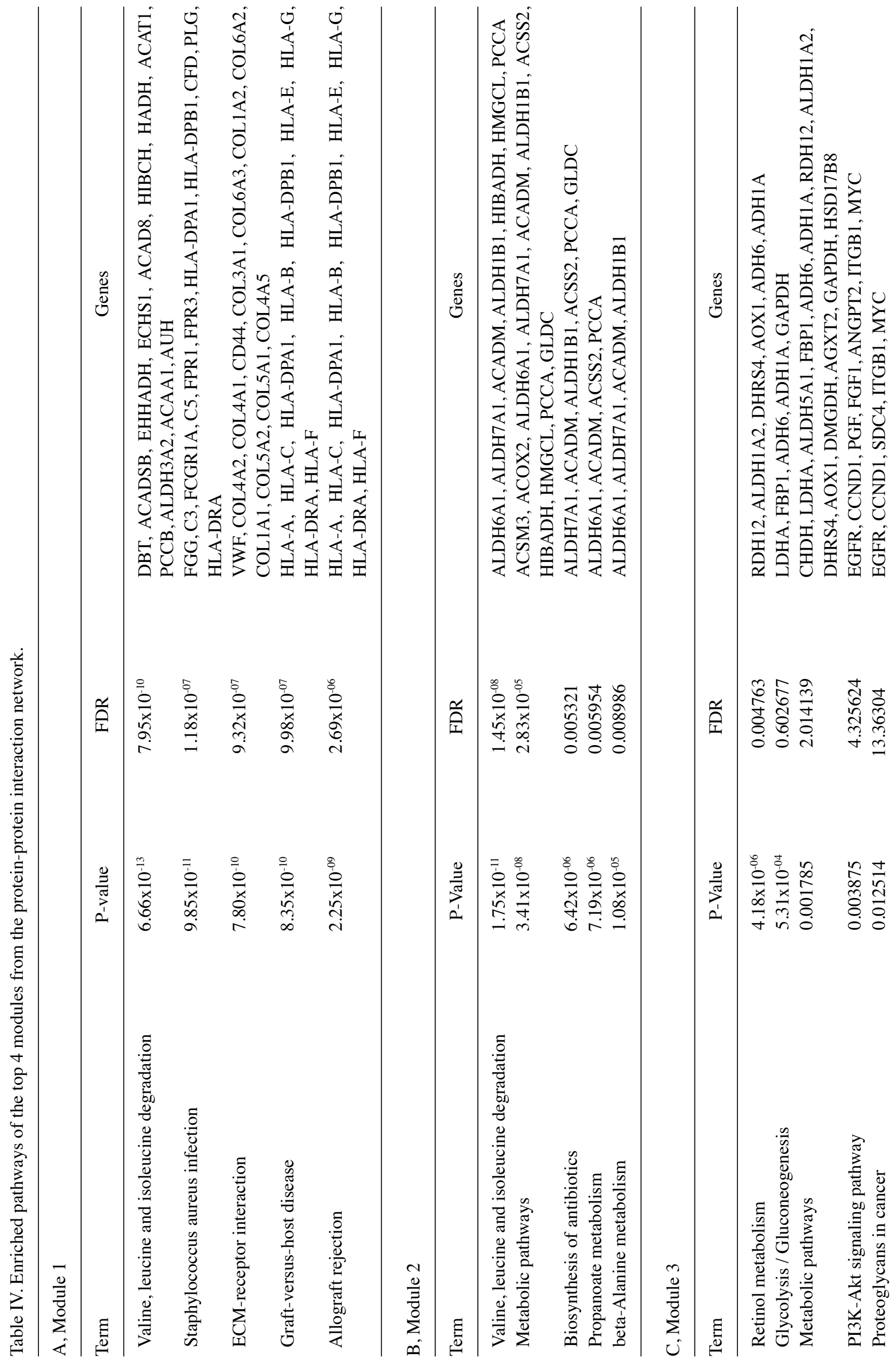




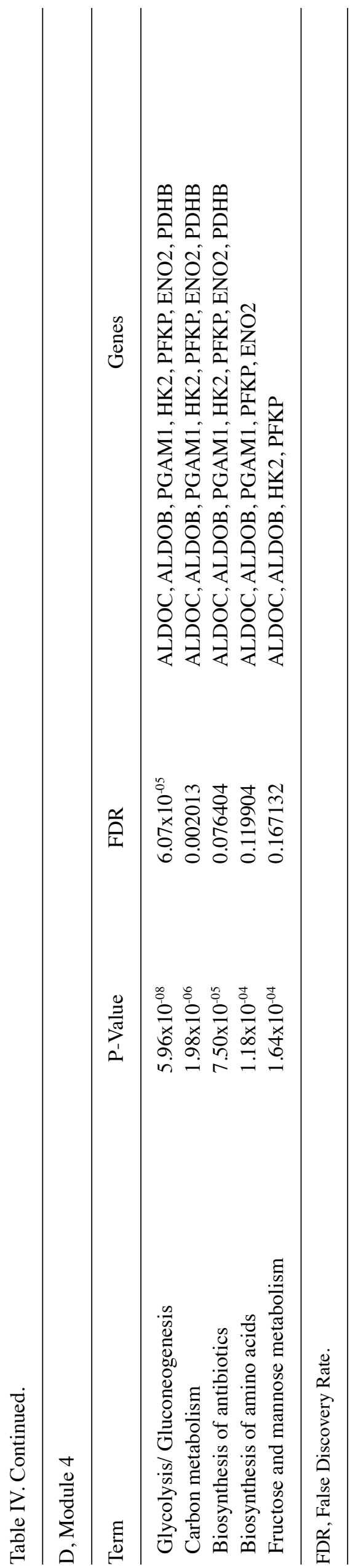

Table V. Relationship between GLDC expression and characteristics of 840 patients with renal cell carcinoma from the Human Protein Atlas.

\begin{tabular}{lccccc}
\hline Characteristics & $\mathrm{n}$ & \multicolumn{2}{c}{ GLDC } \\
& expression & $\chi^{2}$ & P-value \\
\hline Age & & High & Low & 8.223 & 0.00414 \\
$\geq 55$ & 565 & 302 & 263 & & \\
$<55$ & 275 & 118 & 157 & & \\
Sex & & & & 3.108 & 0.07791 \\
$\quad$ Male & 564 & 294 & 270 & & \\
Female & 276 & 126 & 150 & & \\
Stage & & & & 10.229 & 0.00138 \\
$\quad$ I-II & 552 & 298 & 254 & & \\
III-IV & 288 & 122 & 166 & & \\
\hline
\end{tabular}

GLDC, glycine decarboxylase.

silencing inhibiting tumor development, whereas GLDC was reported as a putative tumor suppressor gene involved in tumor progression (34). The present study revealed that GLDC was downregulated in RCC and associated with poor prognosis, consistent with the results observed in gastric cancer. The result of the present study suggested that GLDC may be a tumor suppressor gene in RCC, supported by the fact that high GLDC expression levels inhibited proliferation and promoted apoptosis in RCC. Therefore, GLDC may serve an important role in the diagnosis and therapy of RCC.

To date, three enolase isoenzymes have been identified in mammals, including ENO2. Pathways related to ENO2 are metabolism and hypoxia-inducible factor-1 signaling pathway (38). ENO2 participates in glycolysis and promotes the conversion of b-glycerophosphate to dihydroxyacetone phosphate $(39,40)$. In a previous study, ENO2 was shown to be a pro-survival factor in renal cancer (41). However, to the best of our knowledge, the underlying mechanism of this action has not been clarified. In acute lymphoblastic leukemia, ENO2 was reported to promote cell proliferation, glycolysis and glucocorticoid resistance (42). The mechanism of ENO2 was initially demonstrated in the present study, also demonstrating that high ENO2 expression may be a promising prognostic and diagnostic marker for patients with RCC.

DEGs are candidate diagnostic markers of RCC, with some well-established genes, such as EGFR and VEGF already associated with this disease. The EGFR signaling pathway serves an important role in physiological processes, such as cell growth, proliferation and differentiation. Regulation of the EGFR signaling pathway controls the growth and progression of renal cancer (43). VEGF promotes vascular permeability, extracellular matrix degeneration, vascular endothelial cell migration, proliferation and angiogenesis. Studies have shown that VEGF has a significant impact on the epithelial-mesenchymal transition process in kidney cancer $(44,45)$. UMOD gene is associated with more aggressive clinical and pathological characteristics in RCC (46). Carbonic anhydrase 9 (CA9) is a gene from a family of carbonic anhydrases shown to be a biomarker of ccRCC $(47,48)$, 


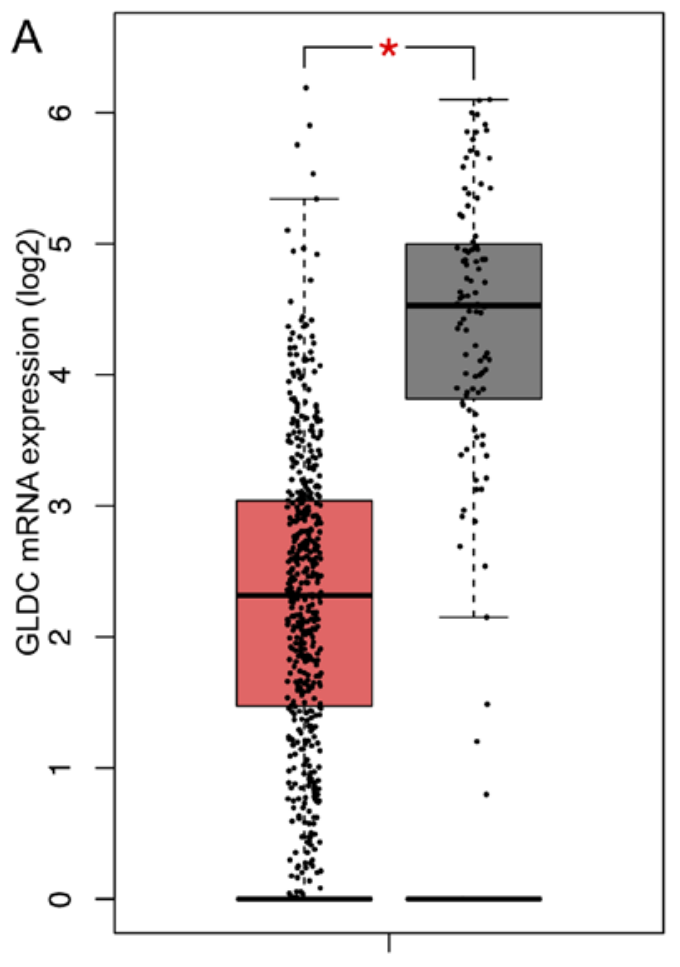

KIRC

(num $(T)=523 ; \operatorname{num}(N)=100)$

C

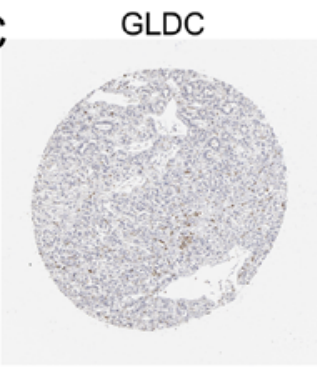

Tumor

$\mathrm{E}$

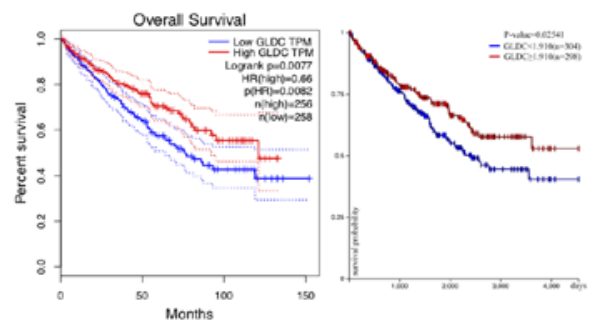

G

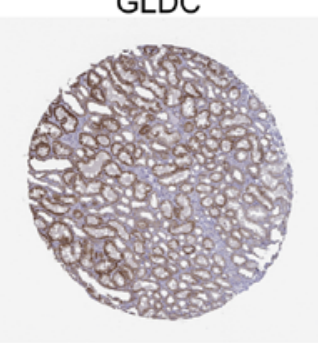

Normal

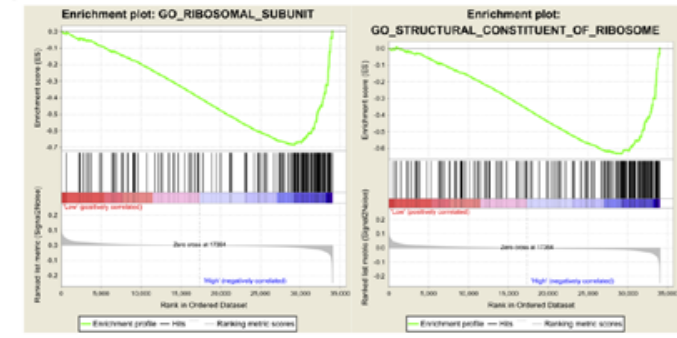

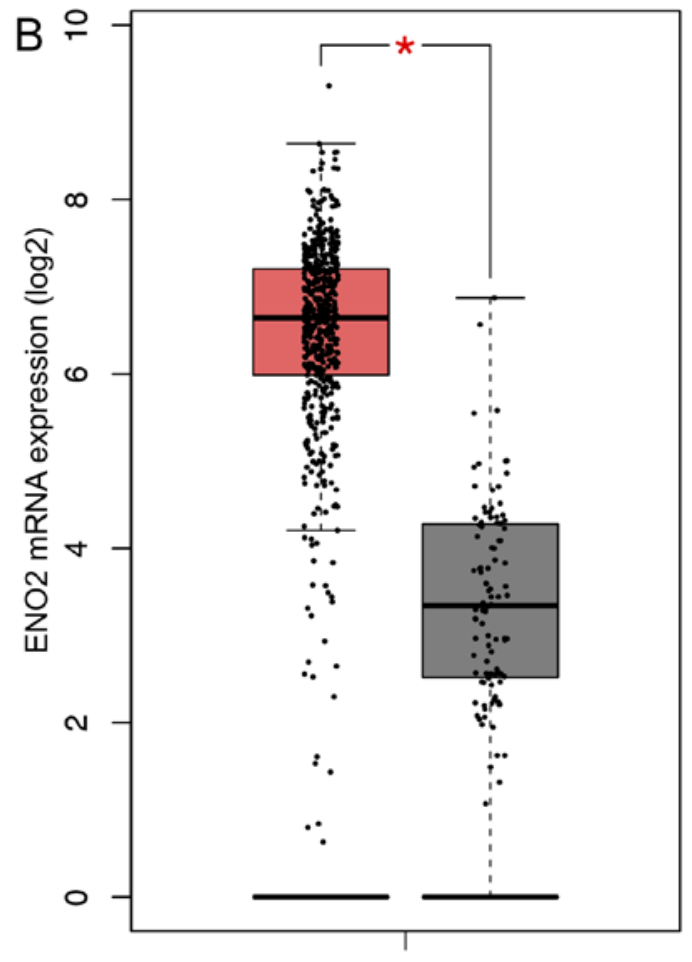

KIRC

(num $(T)=523 ; \operatorname{num}(N)=100$ )

D

ENO2

ENO2

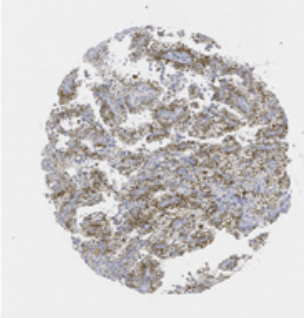

Tumor
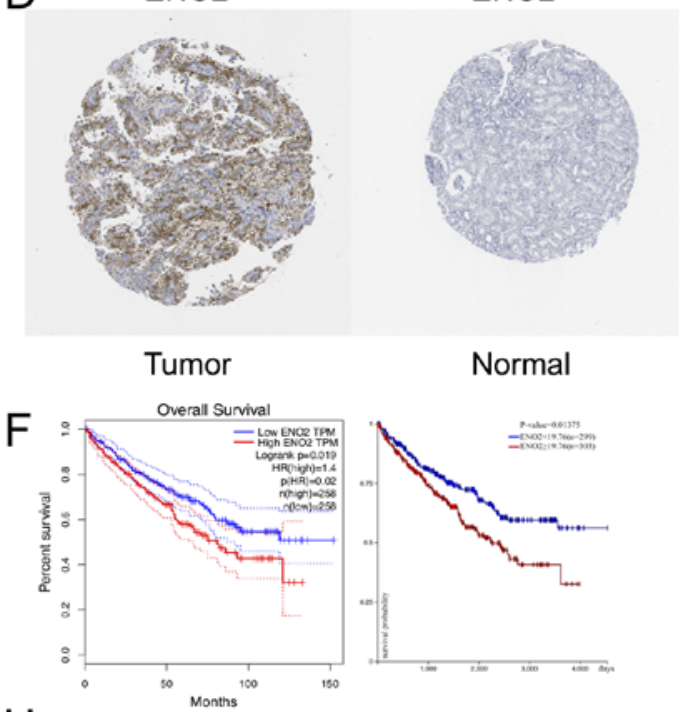

Normal

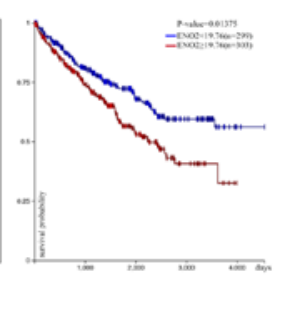

$\mathrm{H}$

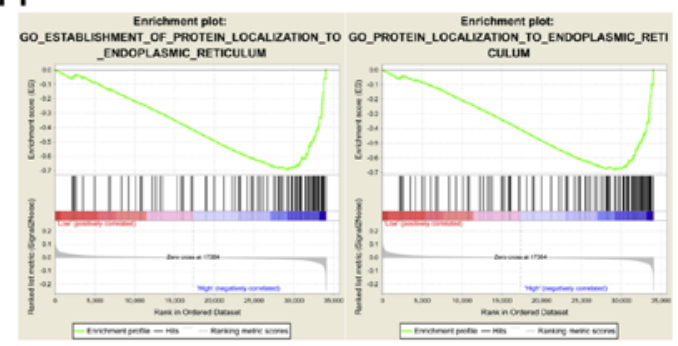

Figure 5. Expression level and GSEA of GLDC and ENO2. Expression levels of (A) GLDC and (B) ENO2 in tumor and nontumor tissues. *P<0.05. (C) GLDC protein expression was downregulated in RCC tissues compared with normal tissues. (D) ENO2 protein expression was significantly upregulated in RCC tissues compared with normal tissues. (E) KM survival curves of GLDC analyzed using GEPIA and UCSC Xena browser. (F) KM survival curves of ENO2 analyzed using GEPIA and UCSC Xena browser. (G) GSEA results showed two significant functional gene sets when GLDC was upregulated in RCC. GeneSet1: GO Ribosomal Subunit, ES=-0.69, Nominal P=0.02. GeneSet2: GO Structural Constituent of Ribosome, ES=-0.63, Nominal P=0.02. (H) GSEA results showed 2 significant functional gene sets when ENO2 was overexpressed in RCC. GeneSet1: GO Establishment of Protein Localization to Endoplasmic Reticulum, Enrichment Score (ES)=-0.69, Nominal P=0.04. GeneSet2: GO Protein Localization to Endoplasmic Reticulum, ES=-0.68, Nominal P=0.02. GLDC, glycine decarboxylase; ENO2, enolase 2; KIRC, Kidney renal clear cell carcinoma; T, tumor; N, normal; HR, hazard ratio; KM; Kaplan Meier; ES, enrichment score; RCC, renal cell carcinoma; GSEA, gene set enrichment analysis; GO, gene ontology. 
A
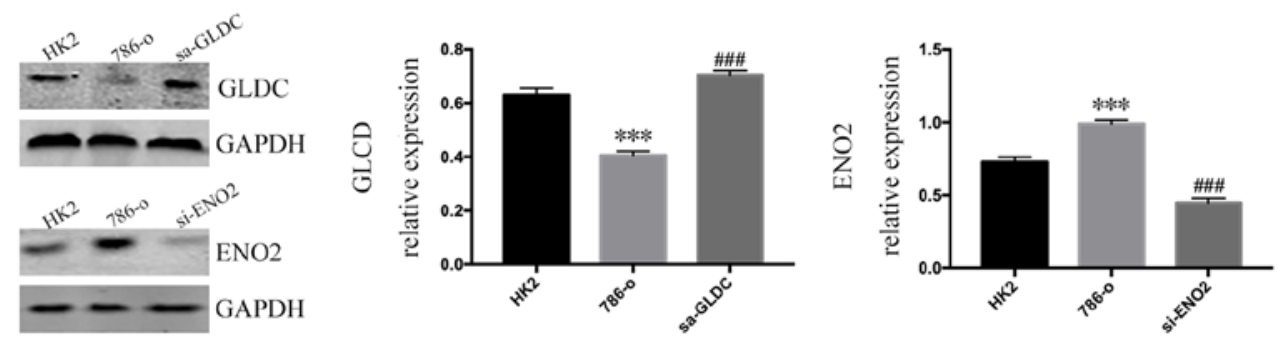

B

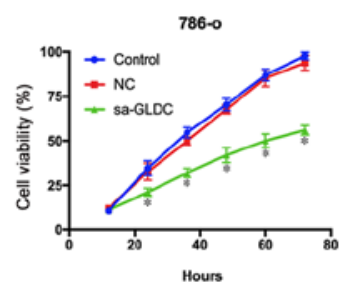

$\mathrm{C}$
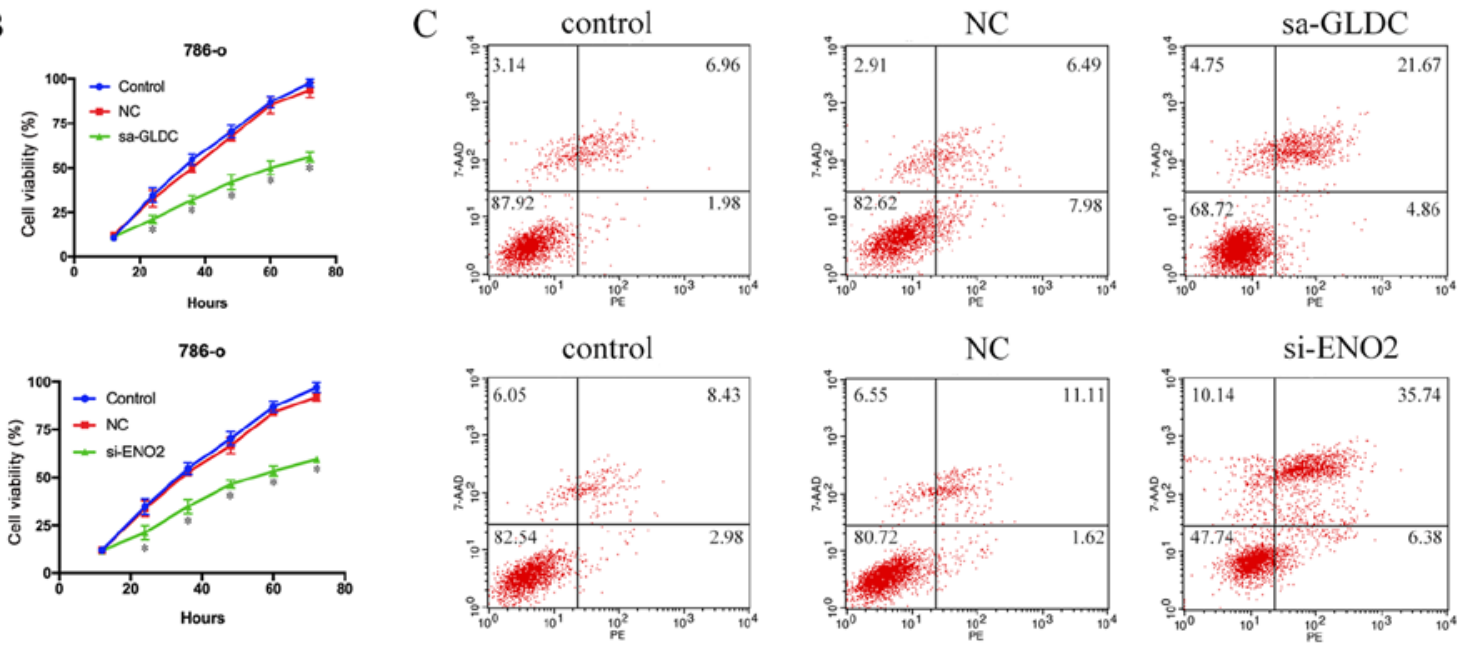

D
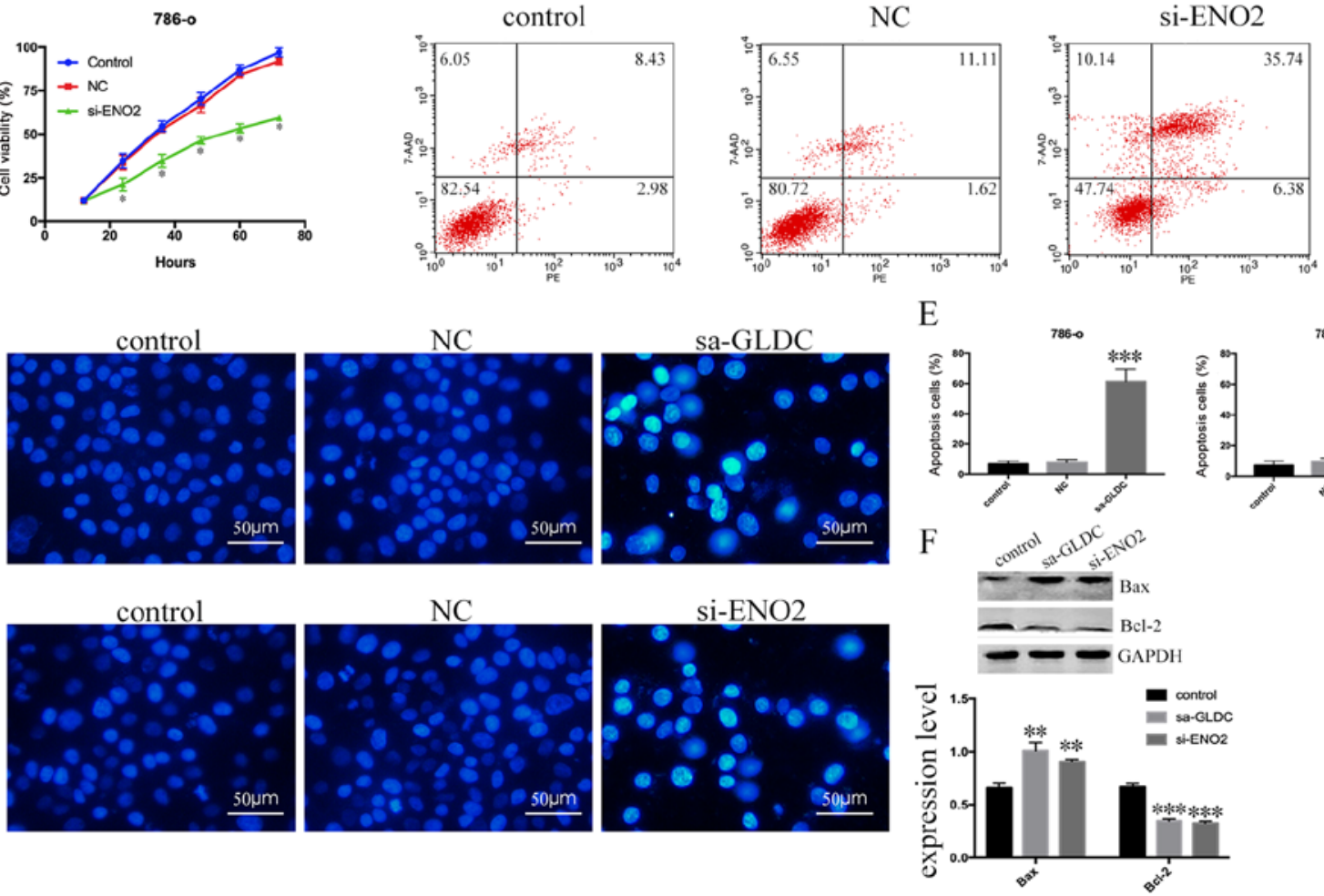

Figure 6. GLDC and ENO2 affected RCC cell apoptosis. (A) Expression of GLDC and ENO2 in HK-2 and 786-O with or without transfection of sa-GLDC or si-ENO2. ${ }^{* * * *} \mathrm{P}<0.001$ vs. HK2; ${ }^{\# \# \# ~} \mathrm{P}<0.0001$ vs.786-O. (B) A Cell Counting Kit-8 assay was performed to determine the proliferative ability of $786-\mathrm{O}$ cells following transfection of sa-GLDC or si-ENO2. ${ }^{*} \mathrm{P}<0.05$ vs. control. (C) Detection of apoptotic rate of 786-O cells using Annexin V-PE/7-AAD double staining. (D) Detection of apoptotic rate of 786-O cells using Hoechst 33258 staining. (E) Quantitative analysis of apoptosis in panel (D). ${ }^{* * *} \mathrm{P}<0.001 \mathrm{vs}$. control. (F) Expression of apoptosis-associated proteins. ${ }^{* *} \mathrm{P}<0.01,{ }^{* * * *} \mathrm{P}<0.001$ vs. Control. Control, untreated $786-\mathrm{O}$ cells; NC, negative control; sa, small activating; si small interfering; GLDC, glycine decarboxylase; PE, phycoerythrin; AAD, 7-aminoactinomycin D.

with previous studies showing that CA9 is overexpressed in RCC (49) and its value as a diagnostic or prognostic marker of ccRCC (50). Pregnancy upregulated nonubiquitous CaM kinase (PNCK) belongs to the calmodulin kinase I family and participates in cytoplasmic and nuclear signal transduction (51). A previous study indicated that overexpression of PNCK could be used for the prognostic stratification of patients with primary ccRCC (52). Collectively, further exploration of the clinical value of these DEGs is warranted.

In the present study, 1,650 DEGs and 15 hub genes were identified as potential diagnostic or prognostic biomarkers of RCC. The present study identified several genes which had not been previously linked with RCC and provided evidence that these genes were associated with this disease. Further experiments are required to verify these results and to more accurately analyze the associations between these genes and RCC. Overall, the present study highlights potentially novel targets for more individualized treatment of patients with RCC.

\section{Acknowledgements}

The authors would like to thank Dr Huan Deng (Department of Gastroenterology, Renmin Hospital of Wuhan University) for his assistance in statistical analysis. 


\section{Funding}

This study was supported by The Application and Basic Research Project of Wuhan City (grant no. 2018060401011321), The Wuhan Morning Light Plan of Youth Science and Technology (grant no. 2017050304010281), The Hubei Province Health and Family Planning Scientific Research Project (grant nos. WJ2017M025 and WJ2017Z005), The Natural Science Foundation of Hubei Province (grant no. 2016CFB114, 2017CFB181) and The Research Project of Wuhan University (grant no. 2042017kf0097).

\section{Availability of data and materials}

The datasets used and/or analyzed during the present study are available from the corresponding author on reasonable request.

\section{Authors' contributions}

$\mathrm{RL}$ and LW performed the biochemical experiments and contributed to the preparation of the draft. XL collected the data and wrote the first draft of the paper. XW perforemd analysis of PPI network. RXG and NL performed the GO and KEGG analysis of hub genes. All authors read and approved the final version of the manuscript.

\section{Ethics approval and consent to participate}

Not applicable.

\section{Patient consent for publication}

Not applicable.

\section{Competing interests}

The authors declare that they have no competing interests.

\section{References}

1. Ferlay J, Soerjomataram I, Dikshit R, Eser S, Mathers C, Rebelo M, Parkin DM, Forman D and Bray F: Cancer incidence and mortality worldwide: Sources, methods and major patterns in GLOBOCAN 2012. Int J Cancer 136: E359-E386, 2015.

2. Patard JJ, Pignot G, Escudier B, Eisen T, Bex A, Sternberg C, Rini B, Roigas J, Choueiri T, Bukowski R, et al: ICUD-EAU international consultation on kidney cancer 2010: Treatment of metastatic disease. Eur Urol 60: 684-690, 2011.

3. Waalkes S, Kramer M. Herrmann TR, Schrader AJ, Kuczyk MA and Merseburger AS: Present state of target therapy for disseminated renal cell carcinoma. Immunotherapy 2: 393-398, 2010.

4. Siegel RL, Miller KD and Jemal A: Cancer statistics, 2018. CA Cancer J Clin 68: 7-30, 2018.

5. Duran I, Lambea J, Maroto P, Gonzalez-Larriba JL, Flores L, Granados-Principal S, Graupera M, Saez B, Vivancos A and Casanovas O: Resistance to targeted therapies in renal cancer: The importance of changing the mechanism of action. Target Oncol 12: 19-35, 2017

6. Gassenmaier M, Chen D, Buchner A, Henkel L, Schiemann M, Mack B, Schendel DJ, Zimmermann W and Pohla H: CXC chemokine receptor 4 is essential for maintenance of renal cel carcinoma-initiating cells and predicts metastasis. Stem Cells 31: 1467-1476, 2013

7. Pan Q, Shai O, Lee LJ, Frey BJ and Blencowe BJ: Deep surveying of alternative splicing complexity in the human transcriptome by high-throughput sequencing. Nat Genet 40: 1413-1415, 2008.
8. Geng RX, Li N, Xu Y, Liu JH, Yuan FE, Sun Q, Liu BH and Chen QX: Identification of core biomarkers associated with outcome in glioma: Evidence from bioinformatics analysis. Dis Markers 2018: 3215958, 2018.

9. Wozniak MB, Le Calvez-Kelm F, Abedi-Ardekani B, Byrnes G, Durand G, Carreira C, Michelon J, Janout V, Holcatova I, Foretova L, et al: Integrative genome-wide gene expression profiling of clear cell renal cell carcinoma in Czech Republic and in the United States. PLoS One 8: e57886, 2013.

10. Davis S and Meltzer PS: GEOquery: A bridge between the gene expression omnibus (GEO) and BioConductor. Bioinformatics 23: 1846-1847, 2007.

11. Thissen D, Steinberg L and Kuang D: Quick and easy implementation of the benjamini-hochberg procedure for controlling the false positive rate in multiple comparisons. J Educ Behav Stat 27: 77-83, 2002.

12. Barrett T, Troup DB, Wilhite SE, Ledoux P, Evangelista C, Kim IF, Tomashevsky M, Marshall KA, Phillippy KH, Sherman PM, et al: NCBI GEO: Archive for functional genomics data sets-10 years on. Nucleic Acids Res 39 (Database Issue): D1005-D1010, 2011.

13. Ashburner M, Ball CA, Blake JA, Botstein D, Butler H, Cherry JM, Davis AP, Dolinski K, Dwight SS, Eppig JT, et al: Gene ontology: Tool for the unification of biology. The gene ontology consortium. Nat Genet 25: 25-29, 2000.

14. Kanehisa M and Goto SA: KEGG: Kyoto encyclopedia of genes and genomes. Nucleic Acids Res 28: 27-30, 2000.

15. Huang da W, Sherman BT and Lempicki RA: Systematic and integrative analysis of large gene lists using DAVID bioinformatics resources. Nat Proto 4: 44-57, 2009.

16. Szklarczyk D, Franceschini A, Wyder S, Forslund K, Heller D, Huerta-Cepas J, Simonovic M, Roth A, Santos A, Tsafou KP, et al: STRING v10: Protein-protein interaction networks, integrated over the tree of life. Nucleic Acids Res 43 (Database Issue): D447-D452, 2015.

17. von Mering C, Huynen M, Jaeggi D, Schmidt S, Bork P and Snel B: STRING: A database of predicted functional associations between proteins. Nucleic Acids Res 31: 258-261, 2003.

18. Shannon P, Markiel A, Ozier O, Baliga NS, Wang JT, Ramage D, Amin N, Schwikowski B and Ideker T: Cytoscape: A software environment for integrated models of biomolecular interaction networks. Genome Res 13: 2498-2504, 2003.

19. Carithers LJ and Moore HM: The genotype-tissue expression (GTEx) project. Biopreserv Biobank 13: 307-308, 2013.

20. Nagy A, Lánczky A, Menyhárt O and Győrffy B: Validation of miRNA prognostic power in hepatocellular carcinoma using expression data of independent datasets. Sci Rep 8: 9227, 2018.

21. Uhlén M, Fagerberg L, Hallström BM, Lindskog C, Oksvold P, Mardinoglu A, Sivertsson Å, Kampf C, Sjöstedt E, Asplund A, et al: Proteomics. Tissue-based map of the human proteome. Science 347: 1260419, 2015.

22. $\mathrm{Li} \mathrm{N}, \mathrm{Li} \mathrm{L}$ and Chen Y: The identification of core gene expression signature in hepatocellular carcinoma. Oxid Med Cell Longev 2018: 3478305, 2018.

23. Subramanian A, Tamayo P, Mootha VK, Mukherjee S, Ebert BL, Gillette MA, Paulovich A, Pomeroy SL, Golub TR, Lander ES and Mesirov JP: Gene set enrichment analysis: A knowledge-based approach for interpreting genome-wide expression profiles. Proc Natl Acad Sci USA 102: 15545-15550, 2005.

24. Pelosi G, Fraggetta F, Nappi O, Pastorino U, Maisonneuve P, Pasini F, Iannucci A, Solli P, Musavinasab HS, De Manzoni G, et al: Pleomorphic carcinomas of the lung show a selective distribution of gene products involved in cell differentiation, cell cycle control, tumor growth, and tumor cell motility: A clinicopathologic and immunohistochemical study of 31 cases. Am J Surg Pathol 27: 1203-1215, 2003.

25. Yan T, Skaftnesmo KO, Leiss L, Sleire L, Wang J, Li X and Enger PO: Neuronal markers are expressed in human gliomas and NSE knockdown sensitizes glioblastoma cells to radiotherapy and temozolomide. BMC Cancer 11: 524, 2011.

26. Murakami N and Riella LV: Co-inhibitory pathways and their importance in immune regulation. Transplantation 98: 3-14, 2014.

27. Zimpfer A, Glass Ä, Zettl H, Maruschke M, Hakenberg OW and Erbersdobler A: Renal cell carcinoma diagnosis and prognosis within the context of the WHO classification 2016. Urologe A 58: 1057-1065, 2019 (In German).

28. Sun C, Yuan Q, Wu D, Meng X and Wang B: Identification of core genes and outcome in gastric cancer using bioinformatics analysis. Oncotarget 8: 70271-70280, 2017. 
29. Chen B, Jiao Z, Yin X, Qian Z, Gu J and Sun H: Novel insights into biomarkers associated with renal cell carcinoma. Oncol Lett 16: 83-90, 2018.

30. Wan B, Huang Y, Liu B, Lu L and Lv C: AURKB: A promising biomarker in clear cell renal cell carcinoma. PeerJ 7: e7718, 2019.

31. Kure S,KatoK,Dinopoulos A,GailC,DeGrauw TJ,Christodoulou J, Bzduch V, Kalmanchey R, Fekete G, Trojovsky A, et al: Comprehensive mutation analysis of GLDC, AMT, and GCSH in nonketotic hyperglycinemia. Hum Mutat 27: 343-352, 2006.

32. Rodriguez-Benitez V, Arranz JA, Mata C, Perez-Navero JL and Gil-Campos M: A new mutation in the GLDC gene in non-ketotic hyperglycinaemia. An Pediatr (Barc) 80: e7-e8, 2014 (In Spanish).

33. Khraim W, Abu-Libdeh B, Ayesh S and Dweikat I: Clinical heterogeneity of glycine encephalopathy in three Palestinian siblings: A novel mutation in the glycine decarboxylase (GLDC) gene. Brain Dev 39: 601-605, 2017.

34. Min HL, Kim J, Kim WH, Jang BG and Kim MA: Epigenetic silencing of the putative tumor suppressor gene GLDC (Glycine Dehydrogenase) in gastric carcinoma. Anticancer Res 36: 179-187, 2016.

35. Kim SK, Jung WH and Koo JS: Differential expression of enzymes associated with serine/glycine metabolism in different breast cancer subtypes. PLoS One 9: e101004, 2014.

36. Berezowska S, Galván JA, Langer R, Bubendorf L, Savic S, Gugger M, Schmid RA and Marti TM: Glycine decarboxylase and HIF-1 $\alpha$ expression are negative prognostic factors in primary resected early-stage non-small cell lung cancer. Virchows Arch 470: 323-330, 2017

37. Zhang WC, Shyh-Chang N, Yang H, Rai A, Umashankar S, Ma S, Soh BS, Sun LL, Tai BC, Nga ME, et al: Glycine decarboxylase activity drives non-small cell lung cancer tumor-initiating cells and tumorigenesis. Cell 148: 259-272, 2012.

38. Zhang T, Niu X, Liao L, Cho EA and Yang H: The contributions of HIF-target genes to tumor growth in RCC. PLoS One 8: e80544, 2013.

39. Isgro MA, Bottoni $P$ and Scatena R: Neuron-specific enolase as a biomarker: Biochemical and clinical aspects. Adv Exp Med Biol 867: 125-143, 2015

40. Vizin T and Kos J: Gamma-enolase: A well-known tumour marker, with a less-known role in cancer. Radiol Oncol 49: 217-226, 2015

41. Luo T, Chen X, Zeng S, Guan B, Hu B, Meng Y, Liu F, Wong T, Lu Y, Yun C, et al: Bioinformatic identification of key genes and analysis of prognostic values in clear cell renal cell carcinoma. Oncol Lett 16: 1747-1757, 2018.

42. Liu CC, Wang H, Wang WD, Wang L, Liu WJ, Wang JH, Geng QR and Lu Y: ENO2 promotes cell proliferation, glycolysis, and glucocorticoid-resistance in acute lymphoblastic leukemia Cell Physiol Biochem 46: 1525-1535, 2018.
43. Laviolette LA, Mermoud J, Calvo IA, Olson N, Boukhali M, Steinlein OK, Roider E, Sattler EC, Huang D, The BT, et al: Negative regulation of EGFR signalling by the human folliculin tumour suppressor protein. Nat Commun 8: 15866, 2017.

44. Huang J, Wang X, Wen G and Ren Y: MiRNA2055p functions as a tumor suppressor by negatively regulating VEGFA and $\mathrm{PI} 3 \mathrm{~K} / \mathrm{Akt} / \mathrm{mTOR}$ signaling in renal carcinoma cells. Oncol Rep 42: 1677-1688, 2019.

45. Roskoski R Jr: Vascular endothelial growth factor (VEGF) and VEGF receptor inhibitors in the treatment of renal cell carcinomas. Pharmacol Res 120: 116-132, 2017.

46. Trevisani F, Larcher A, Cinque A, Capitanio U, Ripa F, Vago R, Bettiga A, Benigni F, Carenzi C, Muttin F, et al: The association of uromodulin genotype with renal cancer aggressiveness. Eur Urol Focus 5: 262-265, 2019.

47. Liao SY, Aurelio ON, Jan K, Zavada J and Stanbridge EJ: Identification of the MN/CA9 protein as a reliable diagnostic biomarker of clear cell carcinoma of the kidney. Cancer Res 57: 2827-2831, 1997.

48. Li G, Cuilleron M, Cottier M, Gentil-Perret A, Lambert C, Genin C and Tostain $\mathrm{J}$ : The use of MN/CA9 gene expression in identifying malignant solid renal tumors. Eur Urol 49: 401-405, 2006.

49. Li G, Cuilleron M, Gentil-Perret A, Cottier M, Passebosc-Faure K, Lambert C, Genin C and Tostain J: Rapid and sensitive detection of messenger RNA expression for molecular differential diagnosis of renal cell carcinoma. Clin Cancer Res 9: 6441-6446, 2003.

50. Li G, Feng G, Gentil-Perret A, Genin C and Tostain J: Serum carbonic anhydrase 9 level is associated with postoperative recurrence of conventional renal cell cancer. J Urol 180: 510-514, 2008.

51. Ueda T, Sakagami H, Abe K, Oishi I, Maruo A, Kondo H, Terashima T, Ichihashi M, Yamamura $\mathrm{H}$ and Minami Y: Distribution and intracellular localization of a mouse homologue of $\mathrm{Ca} 2+/$ calmodulin-dependent protein kinase Ibeta2 in the nervous system. J Neurochem 73: 2119-2129, 1999.

52. Wu S, Lv Z, Wang Y, Sun L, Jiang Z, Xu C, Zhao J, Sun X, Li X, $\mathrm{Hu} \mathrm{L}$, et al: Increased expression of pregnancy up-regulated non-ubiquitous calmodulin kinase is associated with poor prognosis in clear cell renal cell carcinoma. PLoS One 8: e59936, 2013. International (CC BY-NC-ND 4.0) License. 